\title{
OPEN The transcriptional activator of the bfp operon in EPEC (PerA) interacts with the RNA polymerase alpha subunit
}

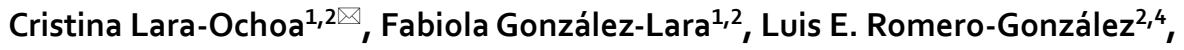 \\ Juan B. Jaramillo-Rodríguez ${ }^{1,3}$, Sergio I. Vázquez-Arellano ${ }^{1}$, Abraham Medrano-López ${ }^{2}$, \\ Lilia Cedillo-Ramírez ${ }^{1}$, Ygnacio Martínez-Laguna ${ }^{1,5}$, Jorge A. Girón ${ }^{1}$, Ernesto Pérez-Rueda ${ }^{6}$, \\ José Luis Puente ${ }^{2 \bowtie}$ \& J. Antonio Ibarra ${ }^{4}$
}

Enteropathogenic $E$. coli virulence genes are under the control of various regulators, one of which is PerA, an AraC/XylS-like regulator. PerA directly promotes its own expression and that of the bfp operon encoding the genes involved in the biogenesis of the bundle-forming pilus (BFP); it also activates PerC expression, which in turn stimulates locus of enterocyte effacement (LEE) activation through the LEE-encoded regulator Ler. Monomeric PerA directly binds to the per and bfp regulatory regions; however, it is not known whether interactions between PerA and the RNA polymerase (RNAP) are needed to activate gene transcription as has been observed for other AraC-like regulators. Results showed that PerA interacts with the alpha subunit of the RNAP polymerase and that it is necessary for the genetic and phenotypic expression of $b f p A$. Furthermore, an in silico analysis shows that PerA might be interacting with specific alpha subunit amino acids residues highlighting the direction of future experiments.

Enteropathogenic Escherichia coli (EPEC) is an E. coli pathovar that causes diarrhea in in children under 5 years of age and elderly people ${ }^{1,2}$. Typical EPEC shows two main virulence-associated phenotypes: 1) forms bacterial aggregates that attach as microcolonies to the host cell surface, a phenotype referred as "localized adherence" (LA) and 2) intimately attaches to the epithelial cells of the small intestine, generating the so-called attaching and effacing (A/E) lesion. Some EPEC strains display only the A/E phenotype and a myriad of adherence phenotypes $^{3,4}$. Both attributes, LA and A/E, depend on two independent protein appendages that are expressed in vivo and in vitro in optimal conditions ${ }^{5,6}$. The LA phenotype is determined by a type IV fimbriae known as the bundle-forming pilus (BFP) encoded in the high-molecular-weight plasmid (pEAF) ${ }^{5,7}$. Formation of the A/E lesion is mediated by the TTSS encoded in the locus of enterocyte effacement (LEE) pathogenicity island ${ }^{1,6}$. BFP biosynthesis depends on the products of 14 genes likely arrayed as an operon, which transcription starts from a promoter upstream of the first gene, $b f p A^{8-10}$. Transcriptional activation of $b f p$ relays on PerA (also known as $\mathrm{BfpT}$ ), a member of the AraC/XylS family of transcriptional activators encoded by the per (per $A B C$ ) operon in the EAF plasmid, which also regulates its own expression ${ }^{11-13}$. The per operon also encodes PerB, which function has not yet been identified, and PerC, which has been shown to activate LEE gene expression through Ler ${ }^{14-16}$ and most recently to regulate EPEC metabolism for nitrate reduction under anaerobic conditions ${ }^{17}$. Thus, as an activator of the genes required for both virulence-related phenotypes, LA and A/E, PerA represents a central regulator of the infectious process caused by typical EPEC strains.

${ }^{1}$ Centro de Detección Biomolecular, Benemérita Universidad Autónoma de Puebla, Puebla, Mexico. ${ }^{2}$ Departamento de Microbiología Molecular, Instituto de Biotecnología, Universidad Nacional Autónoma de México, Cuernavaca, Morelos, Mexico. ${ }^{3}$ Departamento de Ingeniería Celular y Biocatálisis, Instituto de Biotecnología, Universidad Nacional Autónoma de México, Cuernavaca, Morelos, Mexico. "4aboratorio de Genética Microbiana, Departamento de Microbiología, Escuela Nacional de Ciencias Biológicas, Instituto Politécnico Nacional, Mexico City, Mexico. ${ }^{5}$ Vicerrectoría de Investigación y Estudios de Posgrado, Benemérita Universidad Autónoma de Puebla, Puebla, Mexico. ${ }^{6}$ Instituto de Investigaciones en Matemáticas Aplicadas y en Sistemas, Universidad Nacional Autónoma de México, Unidad Académica Yucatán, Mérida, Mexico. ${ }^{\boxplus}$ email: clara_ochoa@hotmail.com; jaig19@gmail.com 
Most of the members of the AraC/XylS family of transcriptional activators have two domains with the following array: the amino terminus, which for some members has a signal-binding and/or dimerization role(s), and the carboxy terminus, which contains the DNA-binding domain where two helix-turn-helix (HTH) motifs are responsible for the DNA binding specificity ${ }^{18-20}$. PerA binds to a conserved sequence located upstream the promoters of the perA and $b f p A$ operons ${ }^{13}$. Critical amino acid residues for PerA function as an activator have been identified at both domains, suggesting that in addition to those required for DNA binding, others seem to bear an important role not involving DNA binding ${ }^{21}$. One possibility is that these residues are involved in interactions with the RNA polymerase (RNAP). Such interactions have been documented in other members of the AraC/XylS family of transcriptional activators ${ }^{22-24}$. In this report we aimed to detect interactions between PerA and the RNAP. Results showed that these interactions occur with the alpha $(\alpha)$ subunit and confirm our previous hypothesis that PerA is a positive regulator that makes specific contacts with the transcriptional machinery and that these interactions are important for the expression of genes under PerA regulation.

\section{Results}

PerA interacts with the RNAP. In order to detect interactions between PerA and the RNAP we first decided to examine whether these contacts occur by using a purified version of PerA. Previously the MBP-PerA fusion allowed us to define this regulator binding sites in both $\operatorname{per} A$ and $b f p A^{13}$. Thus, here we used the MBPPerA fusion to identify interacting proteins in two related experiments: pull downs and co-purifications with cellular extracts of an EPEC strain grown in BFP-A/E inducing conditions. Before performing these experiments, the MBP-PerA purified protein preparation functionality was corroborated by electrophoretic mobility shift assays with the perA regulatory region. Additionally, to corroborate the conditions used to induce the expression of BFP, E2348 WT and $\triangle$ perA mutant strains were grown in inducing and repressing conditions (i.e. by adding ammonium sulfate) and the expression of BfpA was detected by Western blot (Suppl. Fig. 1A). As expected, BfpA was detected only in the WT strain inducing conditions but not under repressing conditions (i.e. DMEM + ammonium salt), nor in the perA mutant strain. MBP-PerA restore BFP expression in a $\triangle$ perA mutant only when no inducer was added (Suppl. Fig. 1B). This latter correlates with our previous observations that when over-produced the MBP-PerA fusion overwhelms the regulatory system and that escape transcription from the lac promoter is enough to produce a small amount of MBP-PerA that ultimately produce the BFP and the LA on tissue cultured cells ${ }^{13}$. In this regard, this small amount of MBP-PerA is not detected by Western blot with the anti-MBP antibodies (Suppl. Fig. 1B). These results showed that the conditions tested induced the expression of BfpA that depends on PerA as previously described ${ }^{9,12,13}$ and that the preparation of purified MBP-PerA was suitable to be used in the following experiments.

Once the experimental system was tested the MBP-PerA fusion was used for pull-down experiments with the cytoplasmic content of $\triangle p e r A$ and WT EPEC strains to detect interacting proteins with this regulator by proteomic analyses. Thus, a pull-down experiment was performed with amylose resin and interacting proteins were resolved by SDS-PAGE (Fig. 1A). Differential protein bands identified between MBP (negative control) and MBP-PerA (bait) pulled down proteins were selected on the basis of being present in the bait but not in the negative control in the three experimental replicates done and their molecular weight; this is, those bands near the expected size for the RNAP subunits were chosen. These bands were excised and analyzed by LC-MS/ MS. In parallel, a co-purification approach was also used and bands were selected using a similar rationale as described above (Fig. 1B). In the co-purification procedure the plasmids encoding either MBP or MBP-PerA (Table 1) were transformed in the $\triangle$ perA EPEC strain and cellular extracts were obtained in inducing conditions. Co-purifed proteins were resolved in an SDS-PAGE and differential bands were identified, selected as described above and submitted for analysis by LC-MS/MS. Among the identified proteins in both experiments the $\beta, \beta$, $\alpha$ and $\sigma$ subunits of the RNAP were identified same as other proteins that will require a further analysis (Table 2 and Suppl. file 1). Taken together, these results suggest that PerA is interacting with the transcriptional machinery.

PerA interacts with the $\alpha$-subunit of the RNAP. Given the results observed with the pull-down and co-purification experiments and that many bacterial transcriptional regulators in the AraC/XylS family make contacts with the alpha $(\alpha)$ subunit of the RNAP ${ }^{18,19,22-26}$, we decided to determine whether PerA interacts with the $a$-subunit of the RNAP (RpoA). First, a purified version of RpoA (His ${ }_{6}$-RpoA) was tested for interactions with MBP-PerA. As seen in Fig. 2, His ${ }_{6}$-RpoA was pulled down with amylose resin and identified by Western blot only when MBP-PerA was used and not with MBP. This result corroborates that both proteins interact in solution in vitro.

In order to further confirm that PerA is interacting with RpoA in vivo two dominant negative versions of RpoA were used. These RpoA negative dominant mutants were described and kindly donated by Dr. Wilma Ross and Dr. Richard Gourse from the University of Wisconsin ${ }^{27,28}$. When over expressed in a bacterium these mutants compete with the wild type RpoA for the rest of the RNAP subunits during its biogenesis. When a defective RpoA is loaded in a RNAP the resulting enzyme is defective for interactions with UP elements in the DNA, but also fails to interact with transcriptional regulators that make contacts with the carboxyl domain of the a-subunit (CTD- $\alpha$ ). Here two RpoA negative dominant mutants lacking different portions of CTD- $\alpha$ encoded in plasmids pLAD235 and pLAD256 (Table 1) were transformed in the wild type EPEC strain and the expression of both perA and $b f p A$ was tested by RT-qPCR from bacterial samples taken from BFP inducing conditions (see above) (Fig. 3). The expression of both genes was observed when an empty vector (pINIIIAa, Table 1) or one encoding a wild type version of $r p o A$ were tested. In contrast, the expression of perA and $b f p A$ was abolished in both RpoA negative dominant mutants.

In order to validate these results, the expression of $\mathrm{BfpA}$ was tested by Western blot in the clones expressing the RpoA negative dominant mutants. As seen in Fig. 4, BfpA was faintly detected in both RpoA mutants. Taken 
A

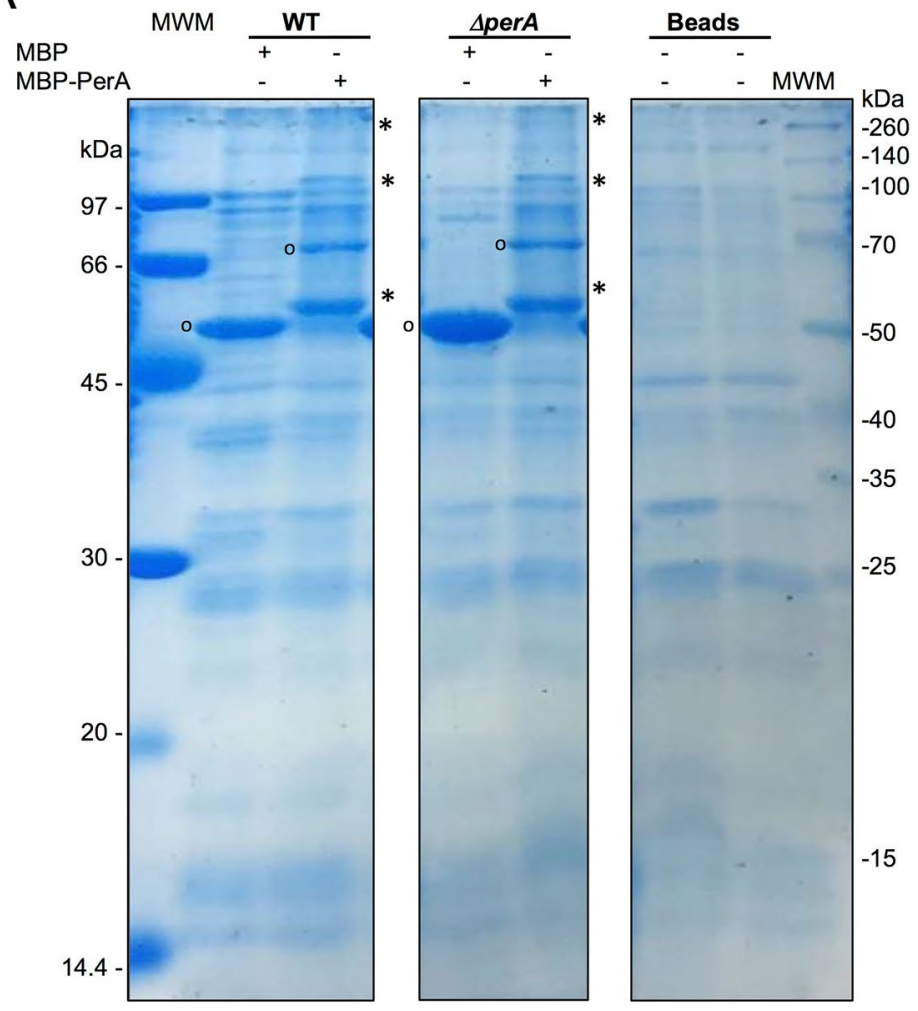

$\mathrm{B}$

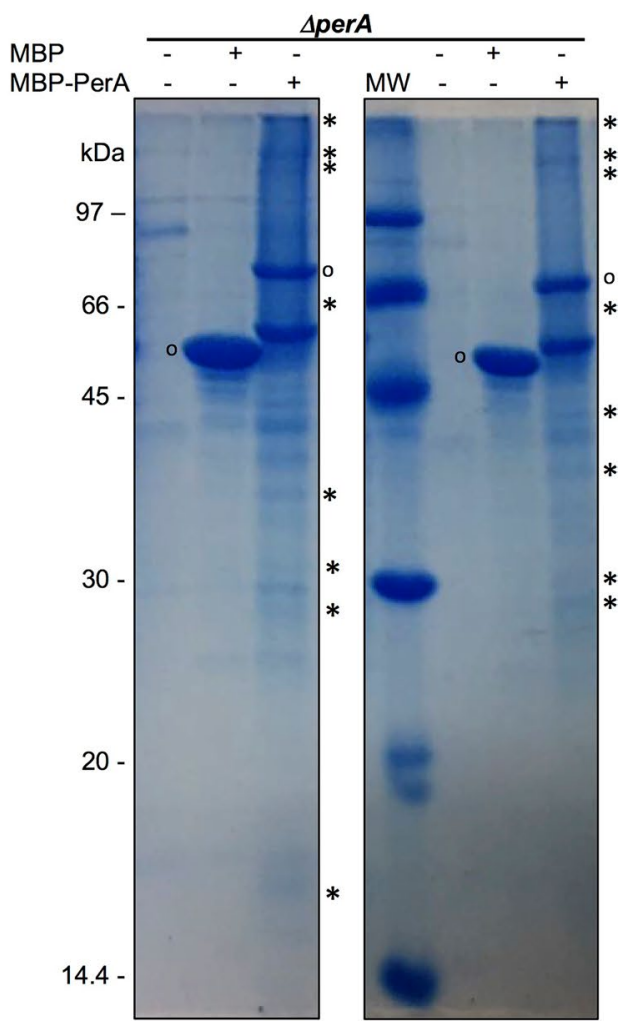

Figure 1. Co-purification and pull-down experiments with MBP-PerA and EPEC strain E2348/69 lysates. Protein-protein interaction experiments for pull-down (A) and co-purification (B) using either purified or over expressed MBP-PerA or MBP were performed as described in the Methods section. Samples were subjected to electrophoresis in a SDS-PAGE and then stained with Coomassie blue. Asterisks indicate proteins that were distinguished as differential when compared with the MBP control and cut for LC-MS/MS analysis and empty circles show the bands corresponding to either MBP or MBP-PerA. Shown are sections of the gels for both pulldown (A) and co-purification (B). Controls for beads are also shown. Experiments were done in triplicate and bands that showed reproducibility were submitted for analysis.

\begin{tabular}{|c|c|c|}
\hline Strain or plasmid & Genotype or description & Source or references \\
\hline \multicolumn{3}{|l|}{ E. coli strain } \\
\hline E2348/69 & Wild-type EPEC O127:H6, Sm $^{\mathrm{R}}$ & 53 \\
\hline JPEP20 & E2348/69 derivate, $\Delta$ perA::kan & 16 \\
\hline MC4100 & F-araD139 $\Delta$ (argF-lac)U169 rpsL150 relA1 flb5301 deoC1 ptsF25 rbsR & 54 \\
\hline BL21(DE3) pLysS & $\begin{array}{l}\text { F-ompT gal dcm lon hsdS }{ }_{B}\left(r_{B}{ }^{-} m_{B}{ }^{-}\right) \lambda(\mathrm{DE} 3[\text { lacI lacUV5-T7p07 ind1sam7 nin5]) } \\
{\left[\text { malB }^{+}\right]_{\mathrm{K}-12}\left(\lambda^{\mathrm{S}}\right) \mathrm{pLysS}\left[T 7 p 20 \text { ori }_{\mathrm{p} 15 \mathrm{~A}}\right]\left(\mathrm{Cm}^{\mathrm{R}}\right)}\end{array}$ & Invitrogen \\
\hline \multicolumn{3}{|l|}{ Plasmids } \\
\hline pMalC2xa & Vector for constructing MBP fusions & New England Biolabs \\
\hline pMALT2 & pMalC2xa derivate expressing MBP-PerA & 21 \\
\hline pET-RpoA & pET28a derivate expressing $\mathrm{His}_{6}$-RpoA & 42 \\
\hline pINIIIA1 & pBR322 derivate cloning vector for mutants of $r p o A$ & 55 \\
\hline pLAX185 & pINIIIA1 derivate expressing wild type RpoA & 27 \\
\hline pLAD235 & $\begin{array}{l}\text { pINIIIA1derivate expressing RpoA truncated from the carboxy end, which retain } 235 \text { amino } \\
\text { acid }\end{array}$ & 27 \\
\hline pLAD256 & $\begin{array}{l}\text { pINIIIAlderivate expressing RpoA truncated from the carboxy end, which retain } 256 \text { amino } \\
\text { acid }\end{array}$ & 27 \\
\hline
\end{tabular}

Table 1. Strains and plasmids used in this study. 


\begin{tabular}{|c|c|c|c|c|c|}
\hline $\begin{array}{l}\text { Estimated molecular weight } \\
(\mathrm{kDa})^{\mathrm{a}}\end{array}$ & Experiment $^{\mathrm{b}}$ & Protein identity ${ }^{c}$ & Molecular weight $(\mathbf{k D a})$ & Accession number (GenBank) & Protein coverage $(\%)^{d}$ \\
\hline \multirow{2}{*}{290} & \multirow{2}{*}{ Co-purification } & $\begin{array}{l}\text { DNA-directed RNA polymerase } \\
\text { subunit beta' }\end{array}$ & 155 & WP_000653936.1 & 75 \\
\hline & & $\begin{array}{l}\text { DNA-directed RNA polymerase } \\
\text { subunit beta }\end{array}$ & 151 & WP_000263098.1 & 56 \\
\hline \multirow{4}{*}{140} & \multirow{4}{*}{ Pull-down } & $\begin{array}{l}\text { DNA-directed RNA polymerase } \\
\text { subunit beta' }\end{array}$ & 155 & WP_000653936.1 & 75 \\
\hline & & $\begin{array}{l}\text { DNA-directed RNA polymerase } \\
\text { subunit beta }\end{array}$ & 151 & WP_000263098.1 & 71 \\
\hline & & $\begin{array}{l}\text { DNA-directed RNA polymerase } \\
\text { sigma factor RpoD }\end{array}$ & 70 & WP_000437371.1 & 44 \\
\hline & & $\begin{array}{l}\text { ATP-dependent Clp protease ATP- } \\
\text { binding subunit ClpA }\end{array}$ & 84 & WP_000934041.1 & 36 \\
\hline \multirow{3}{*}{110} & \multirow{3}{*}{ Pull-down } & $\begin{array}{l}\text { DNA-directed RNA polymerase } \\
\text { subunit beta' }\end{array}$ & 155 & WP_000653936.1 & 41 \\
\hline & & $\begin{array}{l}\text { DNA-directed RNA polymerase } \\
\text { sigma factor RpoD }\end{array}$ & 70 & WP_000437371.1 & 26 \\
\hline & & $\begin{array}{l}\text { DNA-directed RNA polymerase } \\
\text { subunit beta }\end{array}$ & 151 & WP_000263098.1 & 20 \\
\hline \multirow{3}{*}{32} & \multirow{3}{*}{ Pull-down } & $\begin{array}{l}\text { DNA-binding transcriptional } \\
\text { regulator KdgR }\end{array}$ & 30 & WP_001262188.1 & 58 \\
\hline & & $\begin{array}{l}\text { DeoR/GlpR family transcriptional } \\
\text { regulator }\end{array}$ & 28 & WP_001296480.1 & 54 \\
\hline & & $\begin{array}{l}\text { DNA-directed RNA polymerase } \\
\text { subunit alpha }\end{array}$ & 37 & WP_001162094.1 & 20 \\
\hline \multirow{4}{*}{30} & \multirow{4}{*}{ Pull-down } & $\begin{array}{l}\text { DeoR/GlpR family transcriptional } \\
\text { regulator }\end{array}$ & 28 & WP_001296480.1 & 84 \\
\hline & & Transcriptional regulator FNR & 28 & WP_000611911.1 & 28 \\
\hline & & DNA-binding protein H-NS & 16 & WP_001287378.1 & 25 \\
\hline & & $\begin{array}{l}\text { DNA-directed RNA polymerase } \\
\text { subunit alpha }\end{array}$ & 37 & WP_001162094.1 & 21 \\
\hline
\end{tabular}

Table 2. Summary of identified proteins interacting with MBP-PerA. Complete dataset is in Supplementary file $1 .{ }^{a}$ Estimated by mobility in the SDS-PAGE and compared with the molecular weight marker. ${ }^{\mathrm{b}}$ Indicates from which experiments the analyzed band comes from. ${ }^{c}$ Protein identification was carried out by the Proteomics facility using the E. coli protein database (see the Methods section for details). ${ }^{\mathrm{d}}$ The number indicates percentage of coverage of the corresponding protein with the identified peptides.

together these results demonstrate that the interaction of PerA and RpoA is necessary in vivo for the expression of $p e r A$ and $b f p A$.

PerA-RpoA interaction model. Given the results showing that PerA contacts CTD- $\alpha$ we aimed to develop a structural model of this interaction. Using a previous model generated for the PerA DNA binding domain ${ }^{21}$ based on the related protein Rob and the crystal structure reported for the CTD- $\alpha^{29}$ a contact model of the two carboxyl terminal domains was generated (Fig. 5). This docking model suggests that diverse residues could be involved in the interactions between the C-terminal domain of PerA and CTD- $\alpha$. In particular, we identified a cluster of five residues (A308, L312, S313, L318, and M316) in CTD- $\alpha$ that could interact with PerA residues R169, E175, L176, L223, and T227. These residues exhibit a binding free energy between -1.3 and -4.9, and correspond to the top predicted and crystal models used as benchmark datasets. Indeed, these binding free energy suggest these residues have a high possibility for protein-protein interactions.

\section{Discussion}

PerA is a central regulator for typical EPEC strains as it activates genes involved in virulence. In a previous report we have shown that this transcriptional factor is able to bind to the $b f p A$ and $p e r A$ promoter regions as a monomer ${ }^{13}$. Moreover, residues relevant for its function have been identified in both domains of the protein ${ }^{21}$. Here, our working hypothesis was that, PerA being a positive regulator that might be either a class I or a class II regulator binds to DNA and it probably makes contacts with the RNAP in a similar fashion as some members of the AraC/XylS family ${ }^{30,31-36}$. This idea was supported by the following experimental evidences: genes regulated by PerA are not expressed in its absence ${ }^{10,11}$ and that a few PerA point mutants bind to DNA but fail to fully activate transcription ${ }^{21}$. Here, by using two experimental approaches we showed that PerA binds to subunits of the transcriptional machinery when cellular extracts were obtained from inducing conditions. Considering that these approaches yielded several other additional interacting proteins, confirmation of PerA interactions with the RNAP was in need. The a -subunit of the RNAP is not involved in the catalytic activity of this enzyme but is able to contact UP elements and also in making contacts with transcriptional regulators, favoring transcription in both cases ${ }^{26-28,37}$. The dominant negative variants of RpoA together with protein-protein interactions experiments support the initial proposal that PerA and RpoA make contacts, which are needed for activation of 


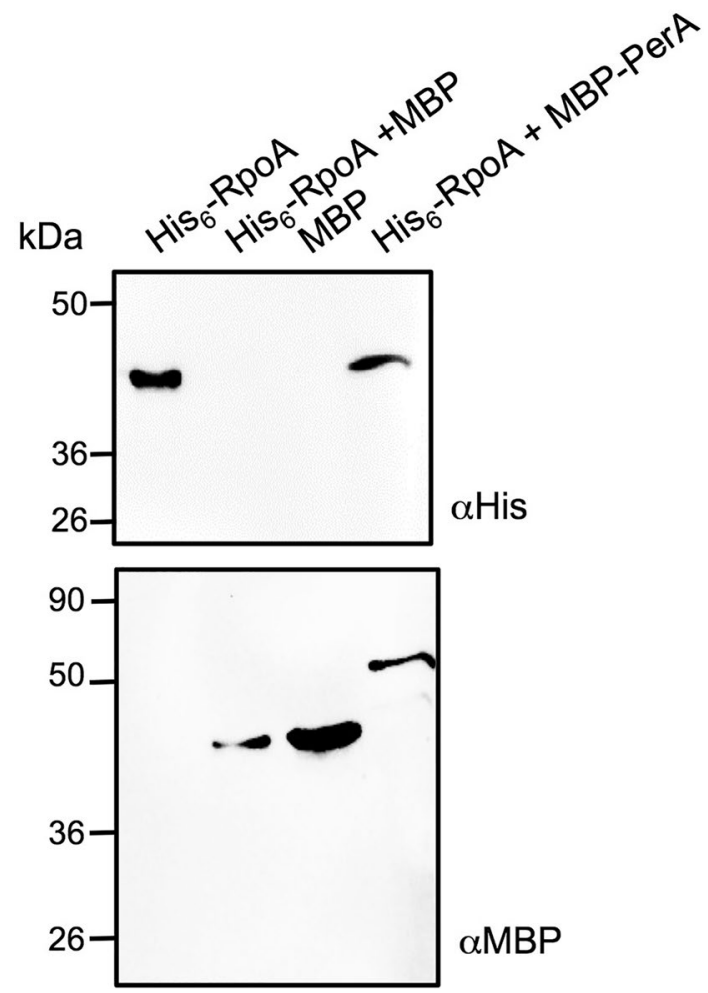

Figure 2. Purified MBP-PerA and $\mathrm{His}_{6}$-RpoA interact in vitro. A pull-down assay was performed with purified $\mathrm{MBP}$ or MBP-PerA proteins and purified $\mathrm{His}_{6}$-RpoA. The mixtures for protein-protein interactions were performed by mixing $50 \mu \mathrm{g}$ of purified MBP or MBP-PerA and $\mathrm{His}_{6}$-RpoA in interaction buffer. To immobilize the bait protein the mixtures were incubated with $50 \mu \mathrm{L}$ of amylose resin. Samples were analyzed by Western blot using an anti-MBP antibody or an anti-His 6 - $\mathrm{HRP}$ probe. In the first lane purified $\mathrm{His}_{6}$-RpoA was used as a control and in the rest of the lanes the interacting mixture is indicated. This figure is a representative of an experiment done in duplicates.

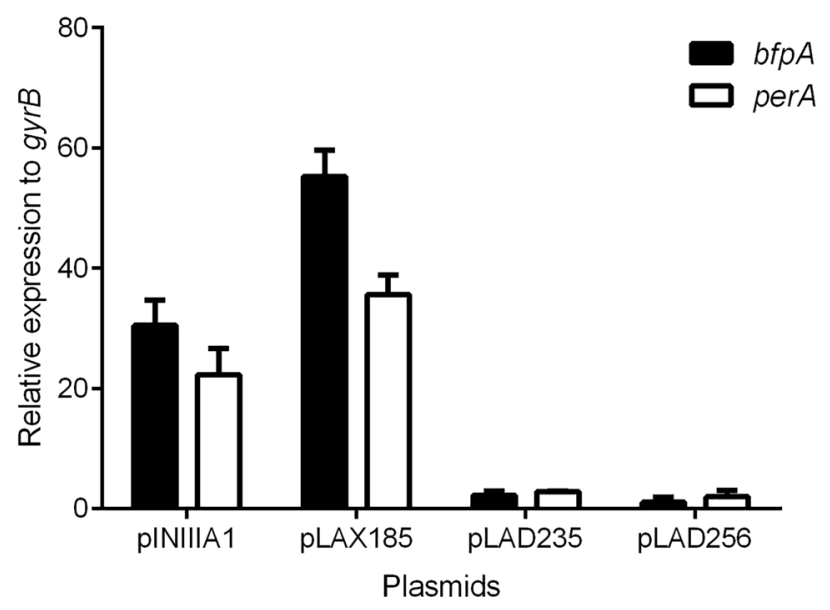

Figure 3. Expression of $b f p A$ and perA is affected in RpoA CTD dominant negative mutants. Transcriptional expression of the $b f p A$ and perA genes in EPEC WT strains transformed with pNIIIA1, pLAX185, pLAD235 and pLAD256 was detected by RT-qPCR. The strains were grown in inducing conditions in DMEM media at $37^{\circ} \mathrm{C}$ supplemented with $0.25 \mathrm{mM}$ IPTG to an D.O ${ }_{600}=0.8$. Graph represents the mean of two independent experiments performed in triplicates, error bars represent the standard error and the bars indicate the average of the relative expression compared to the endogenous genes gyrB. 

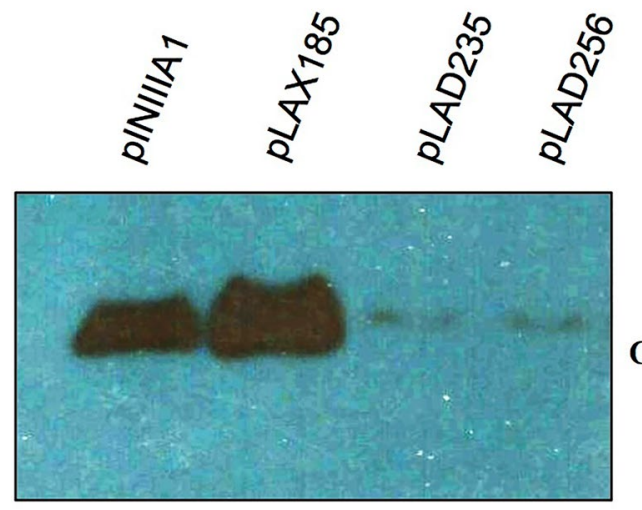

\section{$\alpha B f p A$}

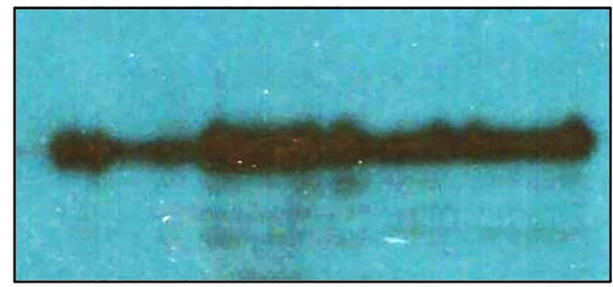

\section{$\alpha G r o E L$}

Figure 4. BfpA expression is defective in RpoA-CTD mutants. BfpA expression in EPEC WT strains transformed with pNIIIA1, pLAX185, pLAD235 and pLAD256 was detected by Western blot in strains grown in DMEM media at $37^{\circ} \mathrm{C}$ in presence of $0.3 \mathrm{mM}$ IPTG at D.O. ${ }_{600}=0.8$. Detection of GroEL was done with antiGroEL antibodies and used as loading and expression controls. This figure is a representative of an experiment done in triplicates.

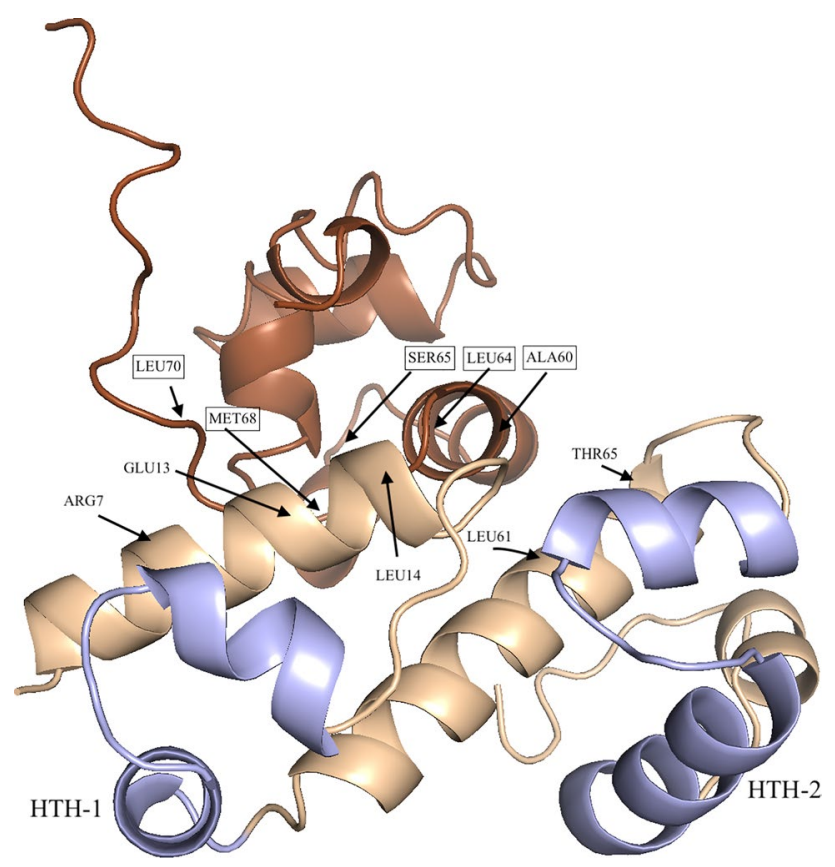

Figure 5. Proposed contacts between PerA and RpoA. A docking model for PerA-CTD and RpoA-CTD was generated the CPHmodels 3.2 server (http://www.cbs.dtu.dk/services/CPHmodels) using the E. coli transcriptional factor Rob (PDB entry 1D5Y chain A) as a template. Stereochemical quality of the model was assessed by using the Ramachandran plot at RAMPAGE program SAVES v6.0 (https://saves.mbi.ucla.edu/). The C-terminal domain of the RNAP a-subunit (PDB entry 1COO) was used to model the interaction with PerA with the HawkDock program (http://cadd.zju.edu.cn/hawkdock). PerA-CTD is shown in gold and purple and RpoA-CTD (CTD- $\alpha$ ) in brown. Putative interacting residues are labeled as follows: RpoA residues are shown in boxes while those from PerA-CTD are not in boxes. PerA HTHs are indicated. 
transcription in vivo. The extent of these interactions as well as residues in each protein that play a role in this interaction will require and direct future experiments in our laboratories.

In the mean time, a 3-D docking model of RpoA and PerA carboxy-terminus domains suggests that two regions in each protein might be making contacts. In RpoA one of these regions is located in the last portion of RpoA (residues 308 to 316). This was an interesting finding as in other transcriptional regulators the so-called "265-determinant" has been shown to be relevant for these interactions. This region has been acknowledged with two roles: 1) Interacting with DNA UP elements and 2) interacting with transcriptional factors ${ }^{22,26}$. In the perA nor in the $b f p A$ regulatory region consensus sequences for UP elements were found, discarding the possibility of RpoA binding to these regulatory regions. Thus, the predicted model suggests that interaction might be occurring in a different region than that previously described, which of course will need experimental assessment. Therefore PerA acts as a positive activator and not as an anti-repressor ${ }^{11,15}$, and it contacts the RNAP through the a-subunit. Studies performed with MarA showed that this regulator makes contacts with the $\alpha$-subunit through the CTD- $\alpha$, specifically with the 265-determinant, in a different manner as CRP 22 . Additionally, it seems that MarA recruits the RNAP to the promoters through a region of 14 amino acid residues in the first $\alpha$-helix $(\alpha-1)$ containing residues that are likely surface exposed and not interacting with the DNA that might be responsible for the MarA-RNAP contacts ${ }^{25}$. These amino acid residues include an aromatic residue in position 14 and aliphatic residues in positions 33 and 37 for MarA. When an alignment between MarA, PerA and other well-characterized members of the family was done we observed that in these positions only V187 (corresponding to V33 in MarA) is conserved in PerA. Our prediction model suggests that PerA residues likely interacting with CTD- $\alpha$ are R169, E175, L176, L223, and T227; the former three are located in a 1 and the rest in $\alpha 4$. In a previous study, when residues E116 and D168 were changed for alanine residues PerA was still able to bind DNA but was differentially affected in its ability to activate perA and $b f p A^{21}$. At that time we suggested that these residues might be involved in interactions with the RNAP. PerA residue D168 is located near the region predicted by the 3-D model and residue $\mathrm{E} 116$ was not detected because resides outside of the $\mathrm{C}$-terminus and therefore is not considered in the modeling. MelR is another member of the AraC/XylS family that has been studied for its contacts with RpoA. For this regulator it has been shown that it makes contact with multiple residues in CTD- $\alpha$ and that they vary depending on which promoter is being activated ${ }^{23}$. Moreover, MelR also makes contact with the $\sigma$-subunit ${ }^{38}$, which is likely to happen also in other members in this family. Lastly, CTD- $\alpha$ interactions have also been shown for other members of the family such as $\mathrm{RhaR}^{39}$, XylS and XylS1 $1^{33,40}$. Thus, despite the fact that the DBD in the AraC/XylS family is widely conserved contacts with the RNAP have not been shown to many of them and we believe that these depend on how each regulator interacts with their corresponding binding site on the DNA. As for PerA, the rest of the residues suggested for the interaction will require further investigation as they represent novel potential sites of contact with CTD- $\alpha$.

In summary, here we have shown that PerA is able to contact the $\alpha$-subunit of the RNAP and that this interaction is necessary for the expression of $b f p A$ and $p e r A$. These results corroborate our previous hypothesis that PerA recruits the RNAP to the promoter regions of these genes.

\section{Methods}

Bacterial strains and culture conditions. The strains and plasmids used in this study are listed in Table 1. Luria-Bertani (LB) broth or Dulbecco's modified Eagle's medium (DMEM) without sodium pyruvate, containing glucose $(0.45 \%)$ and L-glutamine $(584 \mathrm{mg} / \mathrm{L})$ and supplemented with $1 \% \mathrm{LB}(\mathrm{v} / \mathrm{v})$, were used to grow cultures at $37^{\circ} \mathrm{C}$. When indicated the medium was supplemented with ammonium sulfate $(20 \mathrm{mM})$. When necessary, antibiotics were added at the following concentrations: ampicillin $(100 \mu \mathrm{g} / \mathrm{ml})$, streptomycin $(100 \mu \mathrm{g} / \mathrm{ml})$, chloramphenicol $(30 \mu \mathrm{g} / \mathrm{ml})$ and kanamycin $(30 \mu \mathrm{g} / \mathrm{ml})$.

DNA manipulations. DNA manipulations were performed using standard genetic and molecular techniques. Plasmid DNA was purified using High Pure DNA kit (Roche Scientific Inc.). The oligonucleotides used for amplification by PCR were synthesized at T4OLIGO and are listed in Supplementary Table S1. PCR reactions were performed in a $50 \mu \mathrm{l}$ using DreamTaq Green PCR Master Mix (2X) (Thermo Fisher Scientific).

Expression and purification of MBP-PerA and MBP. Expression and purification of the MBP-PerA and MBP proteins was done as described previously ${ }^{13,21,41}$. The concentration of purified proteins was determined by the Bradford method by using an albumin standard curve and analyzed in a $12 \%$ sodium dodecyl sulfate-polyacrilamide (SDS-PAGE) gel electrophoresis. Aliquots were stored at $-70^{\circ} \mathrm{C}$ until used.

Expression and purification of $\mathrm{His}_{6}$-RpoA. The His-tagged RpoA protein was prepared from an E. coli strain BL21 (DE3) containing pET28-RpoA as described previously ${ }^{42}$ (Table 1). Protein concentration was determined by the Bradford method with the use of an albumin standard curve. Aliquots were stored at $-70^{\circ} \mathrm{C}$ until used.

RT-qPCR assays. Relative expression of $b f p A$ and perA in E2348 strains was determined by RT-qPCR as described previously ${ }^{41,43}$. Briefly, RNA was obtained from BFP and PerA inducing conditions described above. DNA was removed with a DNAse I RNAse-free kit (Thermo Fisher Scientific) and cDNA was obtained with a Revert Aid First Strand cDNA Synthesis kit (Thermo Fisher Scientific). qPCR was performed in a Light Cycler 480 Thermocycler (Roche). Relative expression of $b f p A$ and perA was calculated with the $\Delta \Delta \mathrm{Ct}$ method using the expression of the gene coding for the B subunit of the gyrase $(g y r B)$ as a normalizer. Oligos for each gene are listed in Table S1 (bfpA-RTF/bfpA-RTR, perA-RTF/perA-RTR and gyrB-RTF/gyrB-RTR, respectively). Experiments were done in triplicates and the results are the average of two independent experiments. 
Analysis of co-purified proteins. In order to find proteins interacting with PerA the MBP-PerA chimera

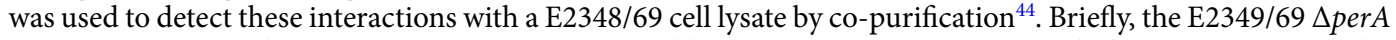
strain containing either pMALT2 or pMALC2xa (Table 1) was grown to exponential phase in DMEM media as described above supplemented with ampicillin $(100 \mu \mathrm{g} / \mathrm{ml})$ and IPTG $(0.3 \mathrm{mM})$. Cultures were pelleted by centrifugation, cells were resuspended in fresh DMEM and subjected to sonication for $10 \mathrm{~min}$, combining 10-s pulses with 10 -s resting cycles in a sonicator. The soluble lysate $(400 \mu \mathrm{l})$ was incubated with amylose magnetics beads $(70 \mu \mathrm{l})\left(\mathrm{New}\right.$ England Biolabs) and let to interact for $6 \mathrm{~h}$ at $4^{\circ} \mathrm{C}$. The beads with bound proteins were washed ten times with a washing buffer $\left(137 \mathrm{mM} \mathrm{NaCl}, 2.7 \mathrm{mM} \mathrm{KCl}, 10 \mathrm{mM} \mathrm{Na}_{2} \mathrm{HPO}_{4}\right.$ and $\left.1.8 \mathrm{mM} \mathrm{KH}_{2} \mathrm{PO}_{4}, \mathrm{pH} 7.4\right)$. The elution of the bounded proteins from the beads was carried out by suspending the pelleted beads in 4X SDS Laemmli sample buffer (10 mM Tris- $\mathrm{HCl}$ [pH 7.4], $200 \mathrm{mM} \mathrm{NaCl}, 1 \mathrm{mM}$ EDTA, $10 \mathrm{mM} \beta$-mercaptoethanol), and heating at $95^{\circ} \mathrm{C}$ for $10 \mathrm{~min}$. Experiments were done in triplicate and bands that showed reproducibility were submitted for analysis as follows: proteins were analyzed by SDS-PAGE and stained with Coomassie blue and protein bands of interest were excised with a new sterile blade for characterization by tryptic digest, liquid chromatography and mass spectrometry analysis (LC-MS/MS) at the Proteomics Discovery Platform of the Institut de Recherches Cliniques de Montréal (Quebec, Canada). Analysis was as done as described before with Mascot (Matrix Science, London, UK; version 2.6.0), and Scaffold (version Scaffold_4.10.0, Proteome Software Inc., Portland, OR). Peptide identifications were accepted if they could be established at greater than $95.0 \%$ probability by the Peptide Prophet algorithm ${ }^{45}$ with Scaffold delta-mass correction. Protein identifications were accepted if they could be established at greater than $95.0 \%$ probability and contained at least 2 identified peptides. Protein probabilities were assigned by the Protein Prophet algorithm ${ }^{46}$. Proteins that contained similar peptides and could not be differentiated based on MS/MS analysis alone were grouped to satisfy the principles of parsimony. Proteins sharing significant peptide evidence were grouped into clusters.

Analysis of pulled down proteins. In order to corroborate interactions of PerA with cytoplasmic proteins from an E2348/69 cell lysate the MBP-PerA was used for pull-down experiments ${ }^{47}$. Purified MBP-PerA or MBP $(40 \mu \mathrm{g})$ were immobilized with $70 \mu \mathrm{l}$ of amylose magnetics beads (New Englands Biolabs) let to interact for $2 \mathrm{~h}$ at $4^{\circ} \mathrm{C}$ and mixed with $400 \mu \mathrm{l}$ of $\Delta$ perA and WT E2348/69 soluble cell extracts for $4 \mathrm{~h}$ in agitation at $4^{\circ} \mathrm{C}$. The beads with bounded proteins were treated as described above. The protein bands of interest were excised for characterization by LC/MS/MS at the Proteomics Discovery Platform of the Institut de Recherches Cliniques de Montréal (Quebec, Canada). Samples were selected from three replicates and analyzed as described in the previous section.

MBP-PerA/His ${ }_{6}$-RpoA interactions (pull down). Pull-down experiments were performed with purified MBP, MBP-PerA and $\mathrm{His}_{6}$-RpoA proteins. Two reaction mixtures were done: A negative control with MBP and $\mathrm{His}_{6}$-RpoA and tests with MBP-PerA and $\mathrm{His}_{6}-\mathrm{RpoA}$; both were done by using $50 \mu \mathrm{g}$ of each protein in a $2 \mathrm{X}$ interaction buffer $\left(100 \mathrm{mM} \mathrm{NaH}_{2} \mathrm{PO}_{4}, 600 \mathrm{mM} \mathrm{NaCl}, 40 \mathrm{mM}\right.$ imidazol, $0.5 \% \mathrm{NP}-40$ and $20 \%$ glycerol, $\mathrm{pH}$ $8.0)^{48}$. Proteins were let to interact for $30 \mathrm{~min}$ on ice, then $50 \mu \mathrm{l}$ of amylose resin or Ni-NTA agarose beads were added to each mixture and let to interact for $2 \mathrm{~h}$ in agitation at $\sim 4^{\circ} \mathrm{C}$. Beads were centrifuged at $2,000 \times g$ for $2 \mathrm{~min}$, amylose beads were washed three times with cold washing buffer and Ni-NTA beads were washed with a low concentration imidazole buffer $(40 \mathrm{mM})$. After the last washing step supernatant was removed carefully and then $20 \mu \mathrm{l}$ of 4 X SDS Laemmli sample buffer were added. Samples were resolved in a 12\% SDS-PAGE and stained with Coomassie blue. Western blot was performed by transferring the gel to a PVDF membrane and by following a previously described protocol with either anti-MBP antibodies (New England Biolabs) or an anti-His6 HRP probe (Invitrogen). Western blot was developed by using chemiluminescence kit (Invitrogen) and observed in a Chemidoc imaging system (Bio-Rad).

Western blotting. For detection of BfpA a sample of $3 \mathrm{ml}$ was taken from bacterial cultures, lysed and the cellular extract used to detect this protein by Western blot. Cells were resuspended in $300 \mu \mathrm{l}$ of a urea solution $(8 \mathrm{M})$ and subjected to sonication. These extracts were combined in Laemmli buffer, boiled, subjected to SDS-PAGE ( $12 \%$ polyacrylamide), and transferred to $0.22-\mu \mathrm{m}$-pore-size PVDF membranes. Membranes were blocked with $10 \%$ nonfat milk and incubated with anti-BfpA $(1: 10,000)$, anti-MBP $(1: 2,000)$ or anti-GroEL $(1: 80,000)$ antibodies. Membranes were then washed with PBS-Tween $20(0.05 \%)$, and a 1:10,000 dilution of horseradish peroxidase-conjugated goat anti-rabbit antibody (Pierce) was added. Membranes were developed by using a SuperSignal West Pico PLUS Chemiluminescent Substrate kit (Thermo Fisher Scientific) according to the manufacturer instructions.

Equipment and settings. Stained SDS-PAGE gels images for Fig. 1 were acquired with a cell phone camera. Western blot images in Fig. 2 were acquired in a ChemiDoc imaging system (Bio-Rad) by using the automatic setting. Later images were converted to a PDF, JPEG or TIFF files with the ImageLab software ver. 6.1 (Bio-Rad). Western blot images in Fig. 4 and Supplementary Fig. 1 were acquired by exposure to a film (Kodak $\mathrm{X}$-Omat LS) and later the selected exposures were either scanned or photographed as mentioned above. Changes in brightness and contrast when considered necessary were done either with the Preview tool in a Mac computer or with Microsoft office picture manager in a Windows computer.

Structural modeling of PerA and the $\alpha$-subunit C-terminal domains. A model of the three-dimensional structure of PerA (P43459) was built with the CPHmodels 3.2 server (http://www.cbs.dtu.dk/services/ CPHmodels) ${ }^{49}$. The E. coli transcriptional factor Rob (PDB entry 1D5Y chain A) was used as a template. To 
refine the model, we used the 3Drefine server (http://sysbio.rnet.missouri.edu/3Drefine/index.html ${ }^{50}$. Finally, for assessing the quality of the protein model, we validated its stereochemical quality of the resulting threedimensional model by using the Ramachandran plot at RAMPAGE SAVES v6.0 program (https://saves.mbi.ucla. $\mathrm{edu} /)^{51}$. The Ramachandran plot, showed that $96.3 \%$ of the residues were located in favored regions and $3.7 \%$ in allowed regions. All the bond distances, angles and dihedrals fulfill the normal limits for polypeptide chains. The resulting model includes 111 of the 274 residues of PerA. The C-terminal domain of the RNAP a-subunit (PDB entry 1COO) was used to model the interaction with PerA. To this end, we used the program HawkDock (http:// cadd.zju.edu.cn/hawkdock), designed to predict protein-protein interactions ${ }^{52}$. The binding free energy of the complex was of $-20.15(\mathrm{kcal} / \mathrm{mol})$, and the top five residues per structure were mapped into the model. Finally, the model was displayed in the PyMOL Molecular Graphics System, Version 2.0 Schrödinger, LLC (https:// pymol.org/).

Statistical analysis. Statistical analysis was performed in Excel or GraphPad Prism version 5 by using a paired-sample Student's $t$-test. A significant difference was considered when $P<0.05$.

Received: 15 November 2020; Accepted: 30 March 2021

Published online: 20 April 2021

\section{References}

1. Kaper, J. B., Nataro, J. P. \& Mobley, H. L. Pathogenic Escherichia coli. Nat. Rev. Microbiol. 2, 123-140. https://doi.org/10.1038/nrmic ro818 (2004).

2. Hu, J. \& Torres, A. G. Enteropathogenic Escherichia coli: foe or innocent bystander?. Clin. Microbiol. Infect. 21(8), 729-734. https:// doi.org/10.1016/j.cmi.2015.01.015 (2015).

3. Tracy, H. H. et al. Refining the pathovar paradigm via phylogenomics of the attaching and effacing Escherichia coli. Proc. Natl. Acad. Sci. USA 110(31), 12810-12815. https://doi.org/10.1073/pnas.1306836110 (2013).

4. Pearson, J. S., Giogha, C., Wong Fok Lung, T. \& Hartland, E. L. The genetics of enteropathogenic Escherichia coli virulence. Annu. Rev. Genet. 50, 493-513. https://doi.org/10.1146/annurev-genet-120215-035138 (2016).

5. Platenkamp, A. \& Mellies, J. L. Environment controls LEE regulation in enteropathogenic Escherichia coli. Front. Microbiol. 9, 1694. https://doi.org/10.3389/fmicb.2018.01694 (2018).

6. Serapio-Palacios, A. \& Finlay, B. B. Dynamics of expression, secretion and translocation of type III effectors during enteropathogenic Escherichia coli infection. Curr. Opin. Microbiol. 54, 67-76. https://doi.org/10.1016/j.mib.2019.12.001 (2020).

7. Giron, J. A., Ho, A. S. \& Schoolnik, G. K. An inducible bundle-forming pilus of enteropathogenic Escherichia coli. Science 254, 710-713 (1991).

8. Sohel, I. et al. Enteropathogenic Escherichia coli: identification of a gene cluster coding for bundle-forming pilus morphogenesis. J. Bacteriol. 178, 2613-2628 (1996).

9. Puente, J. L., Bieber, D., Ramer, S. W., Murray, W. \& Schoolnik, G. K. The bundle-forming pili of enteropathogenic Escherichia coli: transcriptional regulation by environmental signals. Mol. Microbiol. 20, 87-100. https://doi.org/10.1111/j.1365-2958.1996.tb024 91.x (1996).

10. Bustamante, V. H., Calva, E. \& Puente, J. L. Analysis of cis-acting elements required for $b f p A$ expression in enteropathogenic Escherichia coli. J. Bacteriol. 180, 3013-3016 (1998).

11. Martinez-Laguna, Y., Calva, E. \& Puente, J. L. Autoactivation and environmental regulation of $b f p T$ expression, the gene coding for the transcriptional activator of $b f p A$ in enteropathogenic Escherichia coli. Mol. Microbiol. 33, 153-166 (1999).

12. Tobe, T. et al. Complete DNA sequence and structural analysis of the enteropathogenic Escherichia coli adherence factor plasmid. Infect. Immun. 67, 5455-5462 (1999).

13. Ibarra, J. A., Villalba, M. I. \& Puente, J. L. Identification of the DNA binding sites of PerA, the transcriptional activator of the $b f p$ and per operons in enteropathogenic Escherichia coli. J. Bacteriol. 185, 2835-2847 (2003).

14. Gomez-Duarte, O. G. \& Kaper, J. B. A plasmid-encoded regulatory region activates chromosomal eaeA expression in enteropathogenic Escherichia coli. Infect. Immun. 63, 1767-1776 (1995).

15. Porter, M. E. et al. Direct and indirect transcriptional activation of virulence genes by an AraC-like protein, PerA from enteropathogenic Escherichia coli. Mol. Microbiol. 54, 1117-1133 (2004).

16. Bustamante, V. H. et al. PerC and GrlA independently regulate Ler expression in enteropathogenic Escherichia coli. Mol. Microbiol. 82, 398-415. https://doi.org/10.1111/j.1365-2958.2011.07819.x (2011).

17. Mellies, J. L., Platenkamp, A., Osborn, J. \& Ben-Avi, L. PerC Manipulates Metabolism and Surface Antigens in Enteropathogenic Escherichia coli. Front. Cel. Infect. Microbiol. 7, 32. https://doi.org/10.3389/fcimb.2017.00032 (2017).

18. Gallegos, M. T., Schleif, R., Bairoch, A., Hofmann, K. \& Ramos, J. L. Arac/XylS family of transcriptional regulators. Microbiol. Mol. Biol. Rev. 61, 393-410 (1997).

19. Egan, S. M. Growing repertoire of AraC/XylS activators. J. Bacteriol. 184, 5529-5532 (2002).

20. Ibarra, J. A., Perez-Rueda, E., Segovia, L. \& Puente, J. L. The DNA-binding domain as a functional indicator: the case of the AraC/ XylS family of transcription factors. Genetica 133, 65-76 (2008).

21. Ibarra, J. A. et al. Further characterization of functional domains of PerA, role of amino and carboxy terminal domains in DNA binding. PLoS ONE 8(2), e56977. https://doi.org/10.1371/journal.pone.0056977 (2013).

22. Dangi, B., Gronenborn, A. M., Rosner, J. L. \& Martin, R. G. Versatility of the carboxy-terminal domain of the a subunit of RNA polymerase in transcriptional activation: use of the DNA contact site as a protein contact site for MarA. Mol. Microbiol. 54(1), 45-59. https://doi.org/10.1111/j.1365-2958.2004.04250.x (2004).

23. Grainger, D. C., Belyaeva, T. A., Lee, D. J., Hyde, E. I. \& Busby, S. J. Transcription activation at the Escherichia coli melAB promoter: interactions of MelR with the C-terminal domain of the RNA polymerase alpha subunit. Mol. Microbiol. 51(5), 1311-1320. https:// doi.org/10.1111/j.1365-2958.2003.03930.x (2004).

24. Taliaferro, L. P., Keen, E. F. 3rd., Sanchez-Alberola, N. \& Wolf, R. E. Jr. Transcription activation by Escherichia coli Rob at class II promoters: protein-protein interactions between Rob's N-terminal domain and the $\sigma(70)$ subunit of RNA polymerase. J. Mol. Biol. 419(3-4), 139-157. https://doi.org/10.1016/j.jmb.2012.03.019 (2012).

25. Griffith, K. L. \& Wolf, R. E. Jr. A comprehensive alanine scanning mutagenesis of the Escherichia coli transcriptional activator SoxS: identifying amino acids important for DNA binding and transcription activation. J. Mol. Biol. 322, 237-257. https://doi.org/10. 1016/s0022-2836(02)00782-9 (2002). 
26. Haugen, S. P., Ross, W. \& Gourse, R. L. Advances in bacterial promoter recognition and its control by factors that do not bind DNA. Nat. Rev. Microbiol. 6(7), 507-519. https://doi.org/10.1038/nrmicro1912 (2008).

27. Hayward, R. S., Igarashi, K. \& Ishiham, A. Functional specialization within the a-subunit of Escherichia coli RNA polymerase. J. Mol. Biol. 221, 23-29 (1991).

28. Ross, W. et al. Third recognition element in bacterial promoters: DNA binding by the $\alpha$-subunit of RNA polymerase. Science 262, 1407-1413. https://doi.org/10.1126/science.8248780 (1993).

29. Jeon, Y. H. et al. Solution structure of the activator contact domain of the RNA polymerase alpha subunit. Science 270(5241), 1495-1497. https://doi.org/10.1126/science.270.5241.1495 (1995)

30. Browning, D. F. \& Busby, S. J. W. Local and global regulation of transcription initiation in bacteria. Nat. Rev. Microbiol. 14(10), 638-650. https://doi.org/10.1038/nrmicro.2016.103 (2016).

31. Jair, K. W., Fawcett, W. P., Fujita, N., Ishihama, A. \& Wolf, R. E. Jr. Ambidextrous transcriptional activation by SoxS: requirement for the C-terminal domain of the RNA polymerase alpha subunit in a subset of Escherichia coli superoxide-inducible genes. Mol. Microbiol. 19(2), 307-317. https://doi.org/10.1046/j.1365-2958.1996.368893.x (1996).

32. Gillette, W. K., Martin, R. G. \& Rosner, J. L. Probing the Escherichia coli transcriptional activator MarA using alanine-scanning mutagenesis: residues important for DNA binding and activation. J. Mol. Biol. 299, 1245-1255. https://doi.org/10.1006/jmbi.2000. $3827(2000)$.

33. Ruiz, R., Ramos, J. L. \& Egan, S. M. Interactions of the XylS regulators with the C-terminal domain of the RNA polymerase alpha subunit influence the expression level from the cognate Pm promoter. FEBS Lett. 491(3), 207-211. https://doi.org/10.1016/s00145793(01)02192-5 (2001).

34. Shah, I. M. \& Wolf, R. E. Jr. Novel protein-protein interaction between Escherichia coli SoxS and the DNA binding determinant of the RNA polymerase alpha subunit: SoxS functions as a co-sigma factor and redeploys RNA polymerase from UP-elementcontaining promoters to SoxS-dependent promoters during oxidative stress. J. Mol. Biol. 343(3), 513-532. https://doi.org/10.1016/j. jmb.2004.08.057 (2004).

35. Wickstrum, J. R. \& Egan, S. M. Amino acid contacts between sigma 70 domain 4 and the transcription activators RhaS and RhaR. J. Bacteriol. 186(18), 6277-6285. https://doi.org/10.1128/JB.186.18.6277-6285.2004 (2004).

36. Vakulskas, C. A., Brutinel, E. D. \& Yahr, T. L. ExsA recruits RNA polymerase to an extended-10 promoter by contacting region 4.2 of sigma-70. J. Bacteriol. 192(14), 3597-3607. https://doi.org/10.1128/JB.00129-10 (2010).

37. Gaal, T. et al. DNA-binding determinants of the alpha subunit of RNA polymerase: novel DNA-binding domain architecture. Genes \& Dev. 10, 16-26 (1996).

38. Grainger, D. C., Webster, C. L., Belyaeva, T. A., Hyde, E. I. \& Busby, S. J. W. Transcription activation at the Escherichia coli melAB promoter: interactions of MelR with its DNA target site and with domain 4 of the RNA polymerase sigma subunit. Mol. Microbiol. 51(5), 1297-1309. https://doi.org/10.1111/j.1365-2958.2003.03929.x (2004).

39. Holcroft, C. C. \& Egan, S. M. Interdependence of activation at rhaSR by cyclic AMP receptor protein, the RNA polymerase alpha subunit C-terminal domain, and RhaR. J. Bacteriol. 182(23), 6774-6782. https://doi.org/10.1128/JB.182.23.6774-6782.2000 (2000).

40. Ruiz, R. \& Ramos, J. L. Residues 137 and 153 of XylS influence contacts with the C-terminal domain of the RNA polymerase alpha subunit. Biochem. Biophys. Res. Commun. 287(2), 519-521. https://doi.org/10.1006/bbrc.2001.5615 (2001).

41. Romero-González, L. E. et al. The Salmonella Typhimurium InvF-SicA complex is necessary for the transcription of $\operatorname{sop} B$ in the absence of the repressor H-NS. PLoS ONE 15(10), e0240617. https://doi.org/10.1371/journal.pone.0240617 (2020).

42. Rebollar-Flores, J. E. et al. The Salmonella enterica serovar Typhi ltrR gene encodes two proteins whose transcriptional expression is up-regulated by alkaline $\mathrm{pH}$ and repressed at their promoters and coding regions by H-NS and Lrp. J. Bacteriol. 202(13), e00783-e819. https://doi.org/10.1128/JB.00783-19 (2020).

43. Ibarra, J. A. et al. Induction of Salmonella pathogenicity island 1 under different growth conditions can affect Salmonella-host cell interaction in vitro. Microbiology 156(4), 1120-1133. https://doi.org/10.1099/mic.0.032896-0 (2010).

44. Rao, S., Srinivas, G. N. \& Kumar, S. Protein-protein interaction detection: Methods and analysis. Intl. J. Proteomics. 2014, 1-12. https://doi.org/10.1155/2014/147648 (2014).

45. Keller, A., Nesvizhskii, A. I., Kolker, E. \& Aebersold, R. Empirical statistical model to estimate the accuracy of peptide identifications made by MS/MS and database search. Anal. Chem. 74(20), 5383-5392. https://doi.org/10.1021/ac025747h (2002).

46. Nesvizhskii, A. I., Keller, A., Kolker, E. \& Aebersold, R. A statistical model for identifying proteins by tandem mass spectrometry. Anal. Chem. 75(17), 4646-4658. https://doi.org/10.1021/ac0341261 (2003).

47. Louche, A., Salcedo, S. P. \& Bigot, S. Protein-Protein Interactions: Pull-Down Assays. In Bacterial Protein Secretion Systems. Methods in Molecular Biology Vol. 1615 (eds Journet, L. \& Cascales, E.) (Humana Press, 2017).

48. Knodler, L. A., Ibarra, J. A., Perez-Rueda, E., Yip, C. K. \& Steele-Mortimer, O. Coiled-coil domains enhance the membrane association of Salmonella type III effectors. Cell. Microbiol. 13(10), 1497-1517. https://doi.org/10.1111/j.1462-5822.2011.01635.x (2011).

49. Nielsen, M., Lundegaard, C., Lund, O. \& Petersen, T. N. CPHmodels-3.0-remote homology modeling using structure guided sequence profiles. Nucl. Acids Res. 38, W576-W581. https://doi.org/10.1093/nar/gkq535 (2010).

50. Bhattacharya, D., Nowotny, J., Cao, R. \& Cheng, J. 3Drefine: an interactive web server for efficient protein structure refinement. Nucl. Acids Res. 44, W406-W409. https://doi.org/10.1093/nar/gkw336 (2016)

51. Lovell, S. C. et al. Structure validation by Calpha geometry: Phi, psi and Cbeta deviation. Proteins 50(3), 437-450. https://doi.org/ $10.1002 /$ prot.10286 (2003)

52. Weng, G. et al. HawkDock: a web server to predict and analyze the protein-protein complex based on computational docking and MM/GBSA. Nucl. Acids Res. 47(W1), W322-W330. https://doi.org/10.1093/nar/gkz397 (2019).

53. Levine, M. M. et al. The diarrheal response of humans to some classic serotypes of enteropathogenic Escherichia coli is dependent on a plasmid encoding an enteroadhesiveness factor. J. Infect Dis. 152(3), 550-559. https://doi.org/10.1093/infdis/152.3.550 (1985).

54. Casadaban, M. J. Transposition and fusion of the lac genes to selected promoters in Escherichia coli using bacteriophage lambda and Mu. J. Mol. Biol. 104(3), 541-555. https://doi.org/10.1016/0022-2836(76)90119-4 (1976).

55. Masui, Y., Mizuno, T. \& Inouye, M. Novel high-level expression cloning vehicles: $10^{4}$-fold amplification of Escherichia coli minor protein. Nat. Biotechnol. 2, 81-85. https://doi.org/10.1038/nbt0184-81 (1984).

\section{Acknowledgements}

We would like to thank Diana Gómez for the initial steps she did in this project. We are deeply thankful to Dr. Wilma Ross and Dr. Richard Gourse for kindly providing the RpoA negative dominant mutants long time ago. We also appreciate Dr. Liliana Medina-Aparicio (IBT-UNAM) for the gift of plasmid pET-RpoA. Francisco J. Santana and Eugenio López-Bustos from IBT-UNAM are appreciated for their technical assistance. Thanks to Dr. Paulina Estrada-de los Santos for computer pirating. This work was partially supported by grants from Consejo Nacional de Ciencia y Tecnología (CONACYT) awarded to JLP (239659) and JAI (25438).

\section{Author contributions}

The authors C.L.-O. and J.A.I. designed the experiments. C.L.-O, F.G.-L., L.E.R.-G., J.B.J.-R., S.I.V.-A., A.M.-L. and E.P.R. performed the experiments. C.L.-O., J.A.G., Y.M.-L., L.C.-R., J.L.P. and J.A.I. performed the data 
analysis. C.L.-O. and J.A.I. wrote the manuscript. F.G.-L., L.E.R.-G., J.B.J.-R., S.I.V.-A., A.M.-L., E.P.R., J.A.G., Y.M.-L., L.C.-R. and J.L.P. edited the manuscript critically and very carefully. All the authors have read the manuscript and approved the data of manuscript in present form.

\section{Competing interests}

The authors declare no competing interests.

\section{Additional information}

Supplementary Information The online version contains supplementary material available at https://doi.org/ 10.1038/s41598-021-87586-0.

Correspondence and requests for materials should be addressed to C.L.-O., J.L.P. or J.A.I.

Reprints and permissions information is available at www.nature.com/reprints.

Publisher's note Springer Nature remains neutral with regard to jurisdictional claims in published maps and institutional affiliations.

Open Access This article is licensed under a Creative Commons Attribution 4.0 International License, which permits use, sharing, adaptation, distribution and reproduction in any medium or format, as long as you give appropriate credit to the original author(s) and the source, provide a link to the Creative Commons licence, and indicate if changes were made. The images or other third party material in this article are included in the article's Creative Commons licence, unless indicated otherwise in a credit line to the material. If material is not included in the article's Creative Commons licence and your intended use is not permitted by statutory regulation or exceeds the permitted use, you will need to obtain permission directly from the copyright holder. To view a copy of this licence, visit http://creativecommons.org/licenses/by/4.0/.

(C) The Author(s) 2021 